\title{
Anabases
}

ANABASES Traditions et réceptions de l'Antiquité

23 | 2016

Varia

\section{Alexandre à Jérusalem. Variations byzantines sur un thème hérité de Flavius Josèphe}

Corinne Jouanno

\section{OpenEdition}

1 Journals

Édition électronique

URL : http://journals.openedition.org/anabases/5588

DOI : 10.4000/anabases.5588

ISSN : 2256-9421

Éditeur

E.R.A.S.M.E.

Édition imprimée

Date de publication : 2 mai 2016

Pagination : 75-95

ISSN : 1774-4296

\section{Référence électronique}

Corinne Jouanno, «Alexandre à Jérusalem. Variations byzantines sur un thème hérité de Flavius

Josèphe », Anabases [En ligne], 23 | 2016, mis en ligne le 02 mai 2019, consulté le 10 décembre 2020

URL : http://journals.openedition.org/anabases/5588; DOI : https://doi.org/10.4000/anabases.5588

(C) Anabases 


\section{Alexandre à Jérusalem Variations byzantines sur un thème hérité de Flavius Josèphe}

Corinne Jouanno

1 En l'absence d'autres indications, les traductions sont dues à l'auteur du présent article.

Il existe sur cet épisode une très abondante bibliographie: cf. A.Buchler, «La relation de Josèphe concernant Alexandre», REJ 36 (I898), p.I-26; F.PFister, "Eine Gründungsgeschichte Alexandrias und Alexanders Besuch in Jerusalem » [19I4], in Id., Kleine Schriften zum Alexanderroman, Meisenheim am Glan, I976, p. 80 -Io3 (p. 95-103); F.M.AвEL, “Alexandre le Grand en Syrie et en Palestine », RBi 43 (I934), p.528-545 et 44 (I935), p.42-6r; R. Marcus, “Alexander the Great and the Jews », in Id. (éd.), Josephus VI. Jewish Antiquities: Books IX-XI, Cambridge (Mass.), I937, App. C, p.5ı2-532; F. Pfister, "Alexander der Grosse in den Offenbarungen der Griechen, Juden, Mohammedaner und Christen » [I956], inId., KleineSchriften, p.3or-347 (p.320-322); M. Simon, “ Alexandre le Grand Juif et Chrétien », in Id., Recherches d'histoire judéo-chrétienne, Paris - La Haye, ı662, p. I27-i39; A.Momigliano, “Note e discussioni: Flavius Josephus and Alexander's Visit to Jerusalem », Athenaeum n.s. 57 (I979), p.442-448; D. PAcella, "Alessandro e gli Ebrei nella testimonianza dello Ps. Callistene », $A S N P$, ser. III, vol. I2 (I982), p. I255-1269; S.J.D. Cohen, “Alexander the Great and Jaddus the High Priest According to Josephus », Association for Jewish Studies Review 7-8 (I982-I983), p.4I-68; A. Momigliano, “Flavio Giuseppe e la visita di Alessandro a Gerusalemme », in Id., Pagine ebraiche, Turin, I987, p. 87-93; J.Mélèze Modrzejewski, Les Juifs d'Égypte. De Ramsès II à Hadrien, Paris, I99I, p.43-5o; J.A. Goldstein, “Alexander and the Jews », Proceedings of the American 
situe le séjour en question entre la prise des villes de Tyr et de Gaza et le départ du Conquérant pour l'Égypte, sur fond de rivalité entre Juifs et Samaritains²: les Samaritains entreprennent d'emblée de se concilier les faveurs d'Alexandre, en abandonnant la cause de Darius, ce qui leur vaut l'autorisation de bâtir, sur le mont Garizim, un temple destiné à rivaliser avec celui de Jérusalem³; les Juifs, au

Academy for Jewish Research 59 (I993), p.59-Ior; R. Stoneman, "Jewish Traditions on Alexander the Great », StudPhilon 6 (I994), p.37-53 (p.39-43); E. Gruen, Heritage and Hellenism. The Reinvention of Jewish Tradition, Berkeley, I998, p.189-202; M. Sartre, D'Alexandre à Zénobie. Histoire du Levant antique: IV siècle avant J.-C. - III siècle après J.-C., Paris, 200I, p.79-82; T.H. KIм, "The Dream of Alexander in Josephus Ant. xı.325$339 »$, JSJ 34 (2003), p. 425-442; C.H.T. Fletcher-Louis, “Alexander the Great's Worship of the High Priest», in L.T.Stuckenbruck et W.E.S.North (éd.), Early Jewish and Christian Monotheism, Londres - New York, 2004, p. 7I-I02; R.Pummer, The Samaritans in Flavius Josephus, Tübingen, 2009, p. I03-155; A. KLĘCZAR, “The Pagan King before the One God: The Alexander Narrative in Josephus, Antiquitates Iudaicae XI, 8 ", Classica Cracoviensia I5 (2012), p. I37-15o; C. Bonnet, “Alexandre à Jérusalem: entre mensonge et fiction historiographique », in F.M. Simón, F. Pina Polo et J. Remesal Rodríguez (éd.), Fraude, mentiras y engaños en el mundo antiguo, Barcelone, 2014, p.55-64.

2 Bien qu'aucun des historiens du Conquérant ne mentionne un séjour du roi à Jérusalem, on ne peut exclure absolument la possibilité d'une rencontre ou de contacts d'Alexandre avec les Juifs, puisqu'il eut maille à partir avec leurs voisins samaritains : à son départ pour l'Égypte, après la prise de Tyr et de Gaza, il avait en effet confié le gouvernement de la Syrie et de la Palestine à Parménion, qui délégua la charge de la Cœlé-Syrie à Andromachos; celui-ci ayant été brûlé vif par les Samaritains, Alexandre prit des sanctions contre les rebelles à son retour d'Égypte (QCIV, 8, 9). Si l'authenticité de l'épisode a été beaucoup débattue, la thèse de l'anhistoricité domine très largement: Gruen parle d'“outright fabrication» (Heritage, p. I95), SARTre de récits «fabriqués de toutes pièces [...] à des fins de propagande et de polémique », et relevant par conséquent “du mythe d'Alexandre et non de l'histoire»(D’Alexandre à Zénobie, p. 8I).

3 Flavius Josèphe évoque le même type de rivalité entre Juifs et Samaritains émigrés en Égypte, à l'époque hellénistique ( $A J$ XII, IO: trad. NoDET) : “Ils étaient en guerre les uns contre les autres, ceux de Jérusalem affirmant que le Temple qui était chez eux était saint et réclamant qu'on y envoie faire les sacrifices, les Samaritains revendiquant au contraire qu'on aille les faire sur le mont Garizim. » Il relate ensuite assez longuement les démêlés qui opposèrent des représentants des deux peuples, à propos de la sainteté de leurs temples respectifs, à l'époque de PtoléméeVI Philomêtor (I8I-I/45 av. J.-C.) - démêlés arbitrés par le Lagide en personne ( $A J$ XIII, 74-79). Selon toute probabilité, le passage du livreXII constitue une référence anticipée à la dispute racontée au livreXIII (cf. R.EgGer, Josephus Flavius und die Samaritaner, Göttingen, I986, p.95-Ior et 23o-236; Gruen, Heritage, p.240-243; Pummer, The Samaritans, p.I79-I89). Momigliano (“Flavio Giuseppe», p.9o) pense que les légendes juives et samaritaines dont le récit de Josèphe sur Alexandre à Jérusalem se fait l'écho tirent leur origine de 
contraire, parce qu'ils prétendent rester fidèles au pacte qu'ils ont conclu avec le roi perse, s'attirent la colère du Macédonien, qui marche sur Jérusalem, dans un esprit de représailles. Dieu apparaît alors, en rêve, au grand-prêtre Iaddous, pour l'inviter à se porter en grande pompe à la rencontre du Conquérant. Josèphe insiste sur l'effet miraculeux produit sur Alexandre par la solennité de cette procession:

Dès qu'il vit de loin la foule en vêtements blancs, précédée des prêtres habillés de lin, et du grand-prêtre dans son costume couleur d'hyacinthe et tissé d'or, ayant sur la tête la tiare avec la lame d'or où était inscrit le nom de Dieu, Alexandre s'approcha seul, se prosterna devant le Nom et salua en premier le grand-prêtre $\left(A J \mathrm{XI}, 33 \mathrm{I}^{\mathbf{4}}\right)$.

Comme son entourage s'étonne d'un comportement aussi incongru, il explique qu'avant son départ de Macédoine, il a vu en songe Iaddous l'encourageant à partir à la conquête de l'Asie et promettant de lui livrer l'empire des Perses. Le grandprêtre emmène alors Alexandre jusqu'au Temple, où il offre un sacrifice à Dieu ${ }^{5}$ : “On lui montra le Livre de Daniel, où il était annoncé que l'un des Grecs détruirait l'empire des Perses. Il pensa que c'était lui qui était désigné» et «s'en réjouit fort» (AJ XI, 337). La prophétie de Daniel à laquelle Josèphe fait allusion est celle évoquant l'affrontement d'Alexandre et de Darius sous la forme d'un combat entre un bouc et un bélier ${ }^{6}$. Mais, alors que le texte prophétique, composé en réaction aux

ce contexte, et il situe par conséquent l'apparition de l'épisode vers le milieu du II ${ }^{\mathrm{e}}$ s. av. J.-C.; suggestion critiquée par CoHen, qui insiste sur le caractère hétérogène du récit de Josèphe, composé d'éléments de provenances différentes, dont certains, d'origine palestinienne, remonteraient à l'époque pré-maccabéenne («Alexander the Great and Jaddus», p.65-68). Contestant l'hétérogénéité du texte de Josèphe, qu’il qualifie de “well-constructed unit», Goldstein estime pour sa part que l'époque de la cinquième guerre syrienne (203-I97 av. J.-C.), où les Juifs se retrouvèrent tiraillés entre PtoléméeV et Antiochos III, constitue le contexte le plus plausible pour l'émergence de l'épisode jérusalémite, en raison du parallélisme de situation (“Alexander and the Jews », p. 8I et $9 \mathrm{I}-95)$.

4 Trad. E. Nodet, Flavius Josèphe. Les Antiquités juives. Volume V, Livres X et XI, Paris, 2010.

5 Comme le signale Cohen, "Alexander the Great and Jaddus », p.46-49, pareil sacrifice est un élément habituel dans les scènes d'adventus, où l'accomplissement du rite en question désigne le visiteur comme le maître de la cité (Alexandre offrit ainsi un sacrifice à Bel lors de son entrée à Babylone, d'après Arrien, Anab. III, I6, 3-5); mais le contexte dans lequel Josèphe a inséré l'évocation de cet hommage religieux tend à en inverser radicalement la signification.

6 Daniel 8, 5-7 (prophétie) et 20-2I (interprétation): “Le bélier que tu as vu..., c'est le roi des Mèdes et des Perses. Et le bouc [...] est le roi des Grecs. » 
persécutions d'Antiochos IV Épiphane, était animé d'un esprit anti-impérialiste ${ }^{7}$, il n'en va plus de même chez Flavius Josèphe, dont le but est d'insister sur l'amitié qui s'instaure entre Alexandre et le peuple juif. Satisfait des prédictions du grandprêtre, le Conquérant comble de bienfaits les habitants de Jérusalem, auxquels il accorde la liberté d'observer leurs «lois ancestrales», ainsi qu'une exemption d'impôts tous les sept ans ${ }^{8}$. Le récit revient ensuite aux Samaritains qui, jaloux des privilèges accordés aux Juifs, essaient de se faire passer pour tels, afin de bénéficier des mêmes avantages fiscaux - requête à laquelle Alexandre oppose une fin de nonrecevoir. Dans ce long épisode, ce n'est pas le Conquérant qui joue le rôle de héros: servant d'arbitre entre les habitants de Jérusalem et leurs frères ennemis samaritains ${ }^{9}$, il a pour fonction principale de faire valoir le peuple juif, dont les Antiquités

7 Composée $c a$ i65 av. J.-C., sans doute peu de temps avant la mort d'Antiochos IV (advenue en novembre ı64), l'Apocalypse de Daniel est une œuvre de résistance à la conquête (cf. P. Vidal-Naquet, “Les Juifs entre l'État et l'Apocalypse », in C. Nicolet (éd.), Rome et la conquête du monde méditerranéen. II, Genèse d'un empire, $2^{\mathrm{e}}$ éd., Paris, I989, p. 846-882: p. 854 et 88o-882; “Flavius Josèphe et les prophètes », in Id., Les Juifs, la mémoire et le présent, 3 vol., Paris, I99I, t. II, p.2I-5o: p.3I-34; SARtre, D’Alexandre à Zénobie, p.368-369). Dans ses prophéties, Daniel dénonce l’État oppresseur, le royaume grec de Syrie, héritier de l'empire macédonien, dont il souligne d'ailleurs le caractère éphémère (cf. XI, 2-4: «Un roi se lèvera et règnera sur un vaste royaume et fera ce qu'il lui plaît. Tandis qu'il s'élèvera, son royaume sera brisé et partagé aux quatre vents du ciel; il ne conservera pas la même force; il ne sera pas gouverné comme il l'avait gouverné, car son royaume se désagrègera, et cela servira de leçon à d'autres. ») En revanche, dans la paraphrase de Daniel que Josèphe propose au livre $\mathrm{X}$ des Antiquités, toute référence aux limitations de la conquête macédonienne a disparu (cf. X, 273, à propos d'Alexandre [trad. Nodet]: «Le bouc montrait qu'il y aurait un roi issu des Grecs, qui attaquerait deux fois le roi perse, le battrait et s'emparerait de toute sa puissance. »; X, 274, à propos des Diadoques: “Ceux-ci, n’étant ni ses fils ni des proches, gouverneraient le monde habité pendant de nombreuses années. »)

8 Ces mesures d'exemption fiscale rappellent celles consenties aux Juifs par Antiochos III, à l'issue de la cinquième guerre syrienne (202-I95 av. J.-C.): pour les remercier de leur soutien contre les forces lagides, il leur accorda l'autorisation de vivre "selon leurs lois ancestrales", ainsi que des remises d'impôts (Josèphe, $A J$ XII, I/42-I/4). De même, Jules César fit remise aux Juifs, pour l'année sabbatique, des impôts auxquels ils étaient assujettis, en récompense des services qu'ils lui avaient rendus pendant sa campagne d'Égypte, en 48/47 av. J.-C. ( $A J$ XIV, 20I-206).

9 Les Samaritains sont dépeints comme des flagorneurs, prétendant attirer sur eux la protection du Conquérant, alors même que Dieu en réserve l'exclusivité au peuple juif. Sur la présentation, souvent très négative, des Samaritains chez Flavius Josèphe, cf. P.VIdal-Naquet, "Flavius Josèphe ou du bon usage de la trahison", in Flavius Josèphe, La Guerre des Juifs, traduit du grec par P. SAvinel, Paris, I977, p. 7-II5 (p.68-69); 
célèbrent la glorieuse histoire ${ }^{10}$ - Josèphe s'employant ainsi à acquitter une sorte de dette expiatoire à l'égard de la nation qu'il a trahie, quelque trente ans plus tôt, en passant dans le camp des Romains, avant le siège de Jérusalem et la destruction du Temple ${ }^{11}$.

\section{Échos de l'épisode jérusalémite chez les chroniqueurs byzantins}

L'œuvre de Flavius Josèphe a été beaucoup utilisée par les Pères de l'Église ${ }^{12}$, qui lui assurèrent un succès durable tout au long de l'époque byzantine ${ }^{13}$. Aussi

R.J. Coggins, “The Samaritans in Josephus», in L. Feldman et G.Hata (éd.), Josephus, Judaism, and Christianity, Detroit, I987, p. 257-273 (notamment p.26o-263, sur l'épisode jérusalémite); L.F. Feldman, “Josephus’ Attitude Towards the Samaritans : a Study in Ambivalence », in M. Mor (éd.), Jewish Sects. Religious Movements and Political Parties, Creighton, I992, p. 23-45 (notamment p. 29 et 38); Goldstein, “Alexander and the Jews », p. 8I-85; I. HJELm, The Samaritans andEarlyJudaism. ALiterary Analysis, Sheffield, 2000 (notamment p. 204, 208, 222-223, 226-227). Tout en reconnaissant que la présentation des Samaritains est souvent péjorative dans les Antiquités juives (The Samaritans, p. ıo3, II8, I23, I26, I28-I29, I34, I99), Pummer estime toutefois que Flavius Josèphe n'avait pas d'animosité personnelle à leur encontre (ibid., p.64, 27I-285); réserves encore plus marquées chez EgGER, Josephus Flavius und die Samaritaner, p.3ı-3ı6.

10 Cf. Kıм, “The Dream of Alexander », p.439: l'épisode vise à exalter le peuple et la religion juifs plutôt qu'Alexandre; Bonnet, “Alexandre à Jérusalem», p.59: “Flavius Josèphe utilise la geste d'Alexandre pour redorer le blason du peuple élu »; p. 6I : dans son récit, “Alexandre est systématiquement placé en position de demandeur ».

1 Cf. L.H. Feldman, Josephus's Interpretation of the Bible, Berkeley - Los Angeles, I998, p.48: Josèphe «may have felt a responsability to defend the Jews, especially since he himself was under a cloud of suspicion because of his surrender to the Romans in 66. »

12 Cf. H. Schreckenberg, Die Flavius-Josephus Tradition in Antike und Mittelalter, Leyde, I972, p.73-76 (Origène), p.79-88 (Eusèbe), p.9o-9I (Jean Chrysostome), p.98-99 (Théodoret); Id., "The Works of Josephus and the Early Christian Church », in FELdman et Hata (éd.), Josephus..., p. 3ı5-324.

13 Sur la fortune de Flavius Josèphe à Byzance, cf. Schreckenberg, Die Flavius-Josephus Tradition, p. Ior sq.; S.Bowman, "Josephus in Byzantium ", in Feldman et Hata (éd.), Josephus..., p.362-385 (notamment p.377): “The works of Josephus enjoyed the reputation and authority of an ancient encyclopedia whose contents and style were a major influence on the Byzantine perception of its own past.» Schreckenberg dénombre plus de cent vingt manuscrits grecs contenant les œuvres de Flavius Josèphe (Die Flavius-Josephus Tradition, p. I3-47). Bowman précise toutefois que seuls trente-trois de ces manuscrits sont antérieurs au xIV siècle; la popularité de Josèphe semble avoir été particulièrement grande à l'époque tardo-byzantine: plus de la moitié des exemplaires conservés datent de la période des Paléologues (op. cit., p. 368-369). 
l'histoire de la visite d'Alexandre à Jérusalem a-t-elle bénéficié d'une vaste diffusion. Cité dans le Contre Celse d'Origène ${ }^{14}$, dans la Chronique d'Eusèbe de Césarée et dans sa Démonstration évangélique ${ }^{15}$, l'épisode jouissait d'une telle notoriété qu'il est mentionné jusque dans la Topographie chrétienne de Cosmas Indicopleustès $^{16}\left(\mathrm{vi}^{\mathrm{e}} \mathrm{s}\right.$.) et dans les Sacra Parallela, florilège spirituel, transmis sous le nom de Jean Damascène ( $\mathrm{vIII}^{\mathrm{e}} \mathrm{s}$.), où le passage de Josèphe évoquant l'hommage d'Alexandre au grand-prêtre de Jérusalem a été inséré dans le chapitre consacré aux “Bons évêques, qui observent les préceptes de Dieu ${ }^{17}$ ». Mais c'est d'abord et surtout dans les chroniques universelles, genre littéraire très apprécié des Byzantins, que l'épisode jérusalémite rencontra un large accueil. Eusèbe avait donné l'exemple, en mentionnant, dans sa Chronique, l'escale d'Alexandre en Judée et son sacrifice au Dieu des Juifs. Nombreux sont les chroniqueurs qui, à la suite d'Eusèbe, ont évoqué cette visite à Jérusalem dans le chapitre qu'ils consacrèrent au règne du Conquérant ${ }^{18}$ : tel est le cas dans les Excerpta latina Barbari ${ }^{19}$, traduction latine d'une chronique alexandrine dont la composition doit remonter au $\mathrm{V}^{\mathrm{e}}$ siècle, dans la Chronique Pascale (composée entre 63i et 64I), chez Georges le Syncelle (†ap.8Io), Georges le Moine ( $\left.\mathrm{Ix}^{\mathrm{e}} \mathrm{s}.\right)$, Syméon Magistros ( $\left.\mathrm{x}^{\mathrm{e}} \mathrm{s}.\right)$, Georges

14 Contre Celse V, 5o. Sur Origène et Eusèbe, voir W. Mizugaki, “ Origen and Josephus », in Feldman et Hata (éd.), Josephus..., p. 325-337.

15 Chronique, éd. R. Henm, Eusebius Werke. 7. Die Chronik des Hieronymus, Berlin, I956, p.ı23; Démonstration évangélique VIII, 2, 67-68. Sur le rôle d'Eusèbe dans la transmission de Josèphe à Byzance, cf. Bowman, «Josephus in Byzantium », p. 363: «The role of Eusebius is pivotal in the canonization of Josephus as a source for Christian historical identity. ; p.364: “Eusebius' works became the major vehicle through which the Josephan corpus was transmitted to Byzantine chronographers for the next nine centuries. »

16 Topographie chrétienne XII, I4.

17 Sacra Parallela, PG 95, col. ı55oA. L'épisode bénéficie même d'une illustration dans le Parisinus gr. 923, manuscrit du $\mathrm{Ix}^{\mathrm{e}}$ siècle, probablement copié en Palestine (cf. K. Weitzmann, The Miniatures of the Sacra Parallela. Parisinus graecus 923, Princeton, I979, p. 246 et fig. 7I4) : figurant au verso du fol. I92, la miniature représente deux moments du récit de Flavius Josèphe, l'échange verbal d'Alexandre et de Parménion et l'entrée du roi dans le Temple de Jérusalem, sous la conduite du grand-prêtre. Weitzmann précise que la citation des Sacra parallela correspond seulement à la première partie de l'image (entretien d'Alexandre et de Parménion) - preuve que l'illustrateur du Parisinus gr. 923 a reproduit une miniature conçue pour un texte plus complet, relatant sans doute l'intégralité de l'épisode.

18 Sur le traitement réservé au règne d'Alexandre dans les chroniques universelles, voir C.Jouanno, “L'image d'Alexandre le Conquérant chez les chroniqueurs byzantins », Kentron I7/2 (200I), p. 93-ı6.

19 Éd. C. Frick, Chronica Minora, Leipzig, I892, t. I, p. 270 et p.32I-322. 
Kédrénos ( $\left.\mathrm{XI}^{\mathrm{e}}-\mathrm{XII}^{\mathrm{e}} \mathrm{s}.\right)$, Jean Zonaras (†ap. II59) et Michel Glycas $\left(\mathrm{I}^{\mathrm{er}}\right.$ tiers XII ${ }^{\mathrm{e}} \mathrm{s} .-$ - peu av. I204) ${ }^{20}$.

Si les plus anciennes de ces chroniques (Excerpta latina, Chronique Pascale, Georges le Syncelle) ne consacrent que quelques lignes à l'épisode du séjour à Jérusalem, chez Georges le Moine, en revanche, l'étape jérusalémite constitue la pièce maîtresse du développement consacré au Conquérant macédonien : la moitié du chapitre évoquant le règne d'Alexandre est occupée par le récit de cet épisode. Georges le Moine a repris le texte de Flavius Josèphe, qu'il suit de fort près; il a toutefois laissé de côté tout ce qui, dans les Antiquités, concernait les Samaritains, dont il ne souffle mot, et il ne mentionne pas non plus les privilèges fiscaux accordés par Alexandre aux habitants de Jérusalem: c'est sur la signification religieuse de l'épisode qu'il entend mettre l'accent - d'où l'énorme digression qu'il a introduite, au cœur de la narration, pour décrire avec la plus extrême minutie le costume du grand-prêtre, et justifier ainsi l'effet miraculeux produit sur Alexandre par cette tenue qu'irradie la présence divine ${ }^{21}$. Constitué d'un montage de citations tirées de l'Ancien Testament, du livreIII des Antiquités, de plusieurs ouvrages exégétiques de Théodoret et des Erotapokriseis d'Anastase le Sinaïte ${ }^{22}$, cet excursus met clairement en évidence l'enjeu du chapitre sur Alexandre: dans la chronique de Georges le Moine, qui offre une interprétation théologique de l'histoire de l'humanité, Alexandre joue le rôle d'instrument de Dieu, et il est évidemment

20 Chronique Pascale, éd. L. Dindonf, Bonn, i832, t. I, p.39o; Georges le Syncelle, éd. A.A. Mosshammer, Leipzig, ı984, p. 3ı 4 et 3ı 8 ; Georges le Moine, éd. C. DE Boor, Leipzig, I904 (réimpr. I978), t. I, p. 26-32; Syméon Magistros, in Leonis Grammatiki chonographia, éd. I. Bekker, Bonn, I842, p. 49; Georges Kédrénos, éd. I. Bekker, Bonn, I838, t. I, p. 265 et p.27I-272; Jean Zonaras, éd. M. Pinder, Bonn, I84I, t. I, p.329 et 353-355; Michel Glycas, éd. I. BekKer, Bonn, I836, p.267.

21 Longue de 872 mots, cette digression occupe plus de moitié de l'épisode jérusalémite, qui totalise $14 \mathrm{I} 2$ mots.

22 Le point de départ de l'excursus est la description, au chapitre 28 de l'Exode, des vêtements qu'Aaron devra porter dans l'exercice de la prêtrise. À ce texte-fondateur s'ajoutent divers emprunts à I Règnes I4, I8-I9 et 28, 5-7; Flavius Josèphe, $A J$ III, I5I-I87 (notamment § I83); Théodoret, In Exod., questions I5 ( $P G 8 \mathrm{o}$, col. 244A-B) et 6o (PG 8o, col. 284C-285A); In Judic., question I7 (PG 8o, col. 5o5A); In I Reg., question 5 (PG 8o, col. 533D); Anastase le Sinaïte, Quaestiones et responsiones n 98 (éd. M.Richard et J.A. Munitiz, Turnhout, 2006). Dans la Lettre d'Aristée à Philocrate, où figure aussi un passage consacré aux vêtements du grand-prêtre, l'auteur souligne, comme Georges le Moine, la majesté de la tenue sacerdotale, faite pour susciter l'émerveillement: «Le coup d'œil de toute cette pompe en impose et déconcerte, au point de se croire dans un autre monde. Je peux assurer absolument que quiconque ira assister au spectacle dont je viens de parler en sera frappé d'une indicible admiration » ( 7,99 , trad. A. Pelletier, Paris, I962). 
très significatif que le récit de son règne serve de jointure entre la section consacrée à l'histoire profane et celle réservée à l'histoire biblique ${ }^{23}$ : le Conquérant macédonien a, comme l'indiquent les prophéties de Daniel, dûment reproduites par le chroniqueur byzantin, reçu du Ciel la mission de «mettre un terme à l'empire des Perses », et son règne, qui représente une étape importante dans l'histoire du Salut, prépare l'avènement du royaume de Dieu. Georges le Moine a d'ailleurs pourvu l'épisode jérusalémite d'un appendice anecdotique (emprunté au Contre Apion de Flavius Josèphe), qui renforce la signification religieuse de l'ensemble de la séquence: on y voit le Juif Mosomachos, nouvellement enrôlé dans l'armée d'Alexandre, tourner en ridicule un devin qui, sous prétexte d'observer le vol des oiseaux, retardait la marche des troupes macédoniennes, et donner ainsi une leçon à « tous ceux qui étaient asservis à la même erreur ${ }^{24}$ ».

La chronique de Jean Zonaras accorde elle aussi un relief singulier à l'épisode jérusalémite, tout d'abord parce que le séjour d'Alexandre à Jérusalem est mentionné dès le prologue général, dans le passage où Zonaras annonce brièvement le développement qu'il consacrera au règne du Conquérant ${ }^{25}$; ensuite, parce que Zonaras met à nouveau le même épisode en exergue, dans les premières lignes du chapitre dévolu à Alexandre, et en souligne l'intérêt, en le qualifiant de «digne d'admiration » $\left(\theta \alpha u ́ \mu \alpha \tau o \varsigma ~ \alpha ้ \xi ı \nu^{26}\right)$; enfin, parce que le récit de la visite à Jérusa-

23 C'est Alexandre qui a droit au développement le plus détaillé dans la section profane (éd. DE Boor, t. I, p. 6-43) : l'histoire de son règne occupe quatorze pages sur trente-huit, soit plus d'un tiers de cette première section.

24 Éd. DE Boor, t. I, p.32-33. L'anecdote en question est tirée du Contre Apion de Flavius

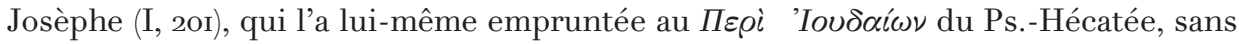
doute composé dans le courant du II $^{\mathrm{e}}$ s. av. J.-C. (FGrH 264, F 2I). C'est la phrase finale du développement consacré dans les Antiquités au séjour d'Alexandre à Jérusalem qui a incité Georges le Moine à greffer l'histoire de Mosomachos sur l'épisode jérusalémite; Josèphe y déclare en effet: “[Alexandre] dit au peuple que, si certains voulaient se joindre à son armée, en conservant les coutumes ancestrales et en en vivant, il était prêt à les emmener; alors beaucoup apprécièrent de faire campagne avec lui » (XI, 339: trad. Nodet).

25 Éd. Pinder, t. I, p. IO-II : “Mais les événements relatifs à Alexandre de Macédoine aussi sont évoqués ici en abrégé: on rappelle, nécessairement, son histoire, entre autres raisons parce que, après la première défaite de Darius à Issos, il séjourna à Jérusalem et accorda au grand-prêtre des honneurs extraordinaires, et [l'on raconte] comment il détruisit la royauté des Perses et les soumit à son pouvoir, combien de temps il régna, et comment son royaume, à sa mort, fut partagé en quatre puissances. »

26 L'histoire du règne d'Alexandre constitue d'ailleurs dans la chronique de Zonaras une sorte d'appendice à celle du peuple juif: c'est après avoir évoqué les relations des Samaritains avec Darius, et la défaite de celui-ci face à Alexandre, que Zonaras ouvre une parenthèse d'une trentaine de pages consacrée au Conquérant macédonien. Cet 
lem constitue l'unique élément hétérogène d'un chapitre qui est, pour le reste, un épitomé de la Vie d'Alexandre de Plutarque ${ }^{27}$. Alors que les autres chroniqueurs des $\mathrm{xI}^{\mathrm{e}}$ et $\mathrm{xII}^{\mathrm{e}}$ siècles, Georges Kédrénos et Michel Glycas, racontent l'épisode jérusalémite en s'inspirant l'un de Georges le Syncelle, et l'autre de Georges le Moine (résumé de façon drastique ${ }^{28}$ ), Zonaras, auteur lettré, est remonté à la source première, Flavius Josèphe, qu'il cite nommément dans l'introduction de son chapitre sur Alexandre ${ }^{29}$ : suivant de fort près le texte des Antiquités, qu'il se contente d'abréger, il est d'ailleurs le seul des chroniqueurs byzantins à faire référence, en fin de chapitre, aux récriminations des Samaritains, jaloux des privilèges accordés aux Juifs ${ }^{30}$.

excursus sur Alexandre est introduit en ces termes (éd. Pinder, t. I, p.329): «Mais puisque mon récit historique a fait référence à Alexandre, il est bon de raconter aussi de façon cursive ( $\left.\varkappa \alpha \tau^{\prime} \varepsilon \dot{\imath} \iota \delta \rho o u n ́ v\right)$ ses actions et ses traits de caractère, de dire où et de qui il était né et de reprendre ensuite la continuité de mon récit; d'autant plus que ce roi séjourna aussi à Jérusalem, qu'il y a accompli à l'égard du grand-prêtre et de son peuple un geste digne d'admiration, et qu'il a lui-même évoqué un rêve inspiré par Dieu, comme le raconte Josèphe. Tout cela, la suite de mon récit l'exposera, après avoir relaté son histoire.»

27 À l'exception d'un bref passage inspiré d'Arrien (Anab. VII, 27), où il est question de la volonté qu'aurait manifestée Alexandre mourant de se jeter dans l'Euphrate, pour faire croire à son apothéose (éd. Pinder, t. I, p.353): c'est précisément ce passage qui fait la soudure entre l'épitomé de Plutarque et l'épisode du séjour à Jérusalem. Après avoir évoqué la mort du Conquérant, le récit de Zonaras renoue avec son point de départ (l'histoire juive), conformément à ce que le chroniqueur avait annoncé dans les quelques lignes d'introduction de l'excursus sur Alexandre (cf. n. 26).

28 Alors que la version de Georges le Moine était longue de I/12 mots, le résumé de Glycas ne compte plus que 83 mots!

29 Sur l'importance de Flavius Josèphe dans la chronique de Zonaras, cf. Bowman, “Josephus in Byzantium», p.37I-372.

30 La version de Zonaras est, au bout du compte, plus fidèle au texte des Antiquités que ne l'était celle de Georges le Moine: il situe la requête initiale d'Alexandre aux Juifs pendant le siège de Tyr et évoque une demande conjointe de renforts militaires et de vivres (cf. $A J$ XI, 3I 7 ); il signale ensuite les craintes du grand-prêtre, son rêve, les consignes que Dieu lui donne - orner la ville et en ouvrir les portes, faire parader le peuple en vêtements blancs (cf. $A J$ XI, 326-327); tous ces détails manquent chez Georges le Moine. Comme Josèphe, Zonaras précise que les compagnons d'Alexandre s'attendent à ce qu'il soumette Jérusalem au pillage et fasse périr le grand-prêtre (cf. $A J \mathrm{XI}, 330$ ); Alexandre indique qu'une vision lui a été accordée alors qu'il se trouvait encore “à proximité de la Macédoine» (cf. $A J$ XI, 334: “à Dion»); l'injonction divine est reproduite au style indirect, comme chez Josèphe, et Zonaras souligne la confiance insufflée à Alexandre par cette apparition (en reprenant la phrase des Antiquités: 
Quelles que soient les libertés prises par certains des chroniqueurs avec la version de Flavius Josèphe, ils lui sont, à une exception près, restés fidèles dans leur évocation de la réaction d'Alexandre face au grand-prêtre: Josèphe parlait d'hommage rendu par le Conquérant au serviteur de Dieu et de sacrifice offert à la divinité elle-même, et l'on retrouve les deux éléments, tantôt séparément, tantôt conjointement, chez nos différents auteurs: Eusèbe, Georges le Syncelle et Kédrénos parlent de sacrifice ${ }^{31}$, la Chronique Pascale et Syméon Magistros de prosternation ${ }^{32}$, Georges le Moine, Zonaras et Glycas de prosternation et de sacrifice $^{33}$. Un seul texte se singularise, en attribuant à Alexandre un véritable acte de foi : il s'agit des Excerpta latina Barbari, où l'on peut lire que le Conquérant « adora le Seigneur Dieu en disant: "Gloire à toi, Dieu unique, maître de toutes choses, qui vis pour les siècles des siècles ${ }^{34 "}$ " - déclaration qui laisse supposer une conversion du roi païen au monothéisme. Une remarque ironique de saint Augustin, dans la Cité de Dieu, suggère que l'auteur de la chronique alexandrine dont dérivent les Excerpta n'était peut-être pas seul à avoir franchi le pas en christianisant Alexandre; Augustin écrit en effet à propos des adversités subies par le peuple juif à l'époque du Second Temple:

“Je pense que c'est une inspiration divine qui a décidé mon expédition...», passage que Georges le Moine avait laissé de côté); l'évocation du sacrifice précède celle de la lecture de Daniel, comme chez Josèphe ( $A J$ XI, 336-337); dans la prophétie, il est question des “Grecs», et non des “Macédoniens», comme chez Georges le Moine; après avoir évoqué très brièvement la générosité du Conquérant à l'égard des Juifs, Zonaras enchaîne sur les requêtes des Samaritains (cf. AJ XI, 340-345).

31 Eusèbe, éd. Helm, p. 123: Deo victimas immolat; Georges le Syncelle, éd. Mosshammer,

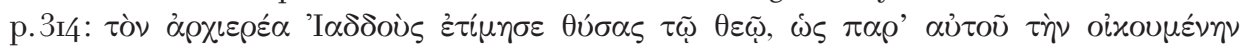

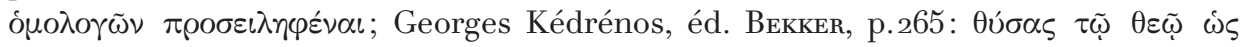

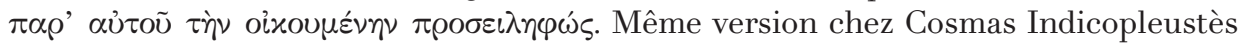
(Topographie chrétienne XII, I4).

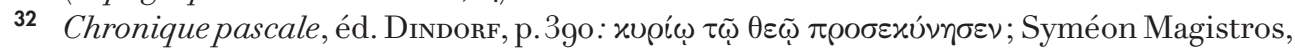

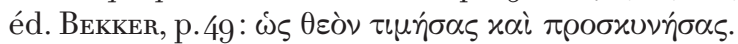

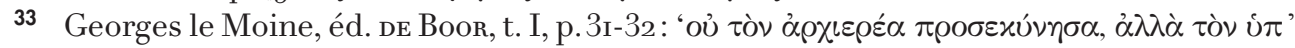

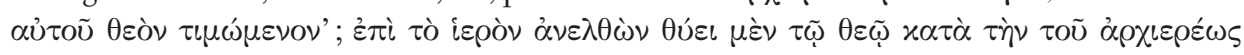

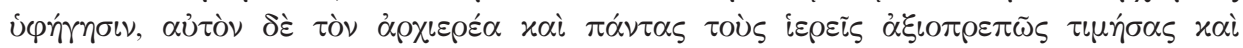

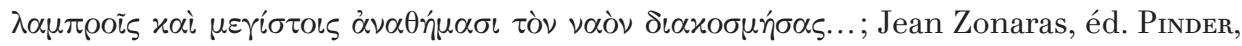

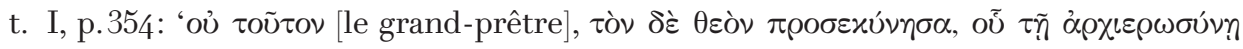

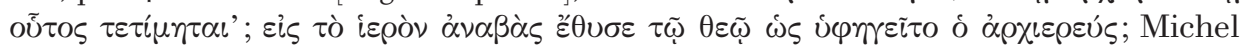

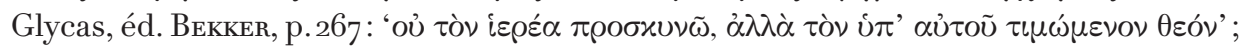

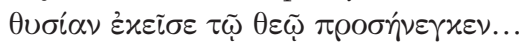

34 Éd. Frick, t. I, p. 270: Domino deo adoravit dicens: “Gloria tibi, deus solus omnia tenens, qui vivis in saecula. »; le même épisode est évoqué une seconde fois, en termes similaires, p. 320 . 


\begin{abstract}
Alexandre survint et subjugua [la nation juive], et bien que cela se soit fait sans dévastation, car les Juifs n'osèrent pas lui résister, et leur prompte soumission apaisa le vainqueur, pourtant la gloire de cette maison fut moindre que sous le libre règne des rois. Sans doute Alexandre immola des victimes dans le temple de Dieu, non qu'il se fût converti à son culte par une véritable piété, mais en sa vanité impie, il pensait devoir l'adorer avec ses faux dieux ${ }^{35}$.

Les chroniqueurs byzantins n'ont pas suivi la voie ouverte par les Excerpta: leur Alexandre apparaît certes comme un instrument de la volonté divine - instrument docile et même reconnaissant -, mais pas comme un confesseur de la foi. À Byzance, seule la littérature de fiction a osé mettre en scène la mutation religieuse du Conquérant macédonien, que l'on voit s'accomplir dans plusieurs réécritures médiévales du Roman d'Alexandre, ce best-seller de la tradition populaire ${ }^{36}$.
\end{abstract}

\title{
Adaptations romanesques de l'épisode d'Alexandre à Jérusalem
}

La publicité que le récit des chroniqueurs apporta à l'épisode jérusalémite explique sans doute que le séjour à Jérusalem ait été inséré dans la plupart des versions byzantines du Roman d'Alexandre, alors que cet épisode était inconnu des plus anciennes recensions du Pseudo-Callisthène, tardo-antique $(\alpha)$ et protobyzantine $(\beta)^{37}$. C'est au tournant du viII ${ }^{\mathrm{e}}$ et du $\mathrm{Ix}^{\mathrm{e}}$ siècle, donc à l'époque de la crise iconoclaste (726-787 et 8I5-843), que cette séquence à contenu fortement religieux fait son entrée dans la tradition pseudo-callisthénienne, dans la recension $\varepsilon$. On la retrouve ensuite dans la recension $\gamma$ (dont l'auteur a simplement reproduit le texte d'ع), puis dans le Poème du Marcianus gr. 408 (a. I388?), dans la recension $\zeta$

35 Cité de Dieu XVIII, 45 (trad. G. Bardy et G. Combes, Paris, I959). Passage signalé par L. Angliviel, “Alexandre le Grand au rv siècle apr. J.-C.: entre païens et chrétiens », Mètis I (2003), p.27I-288 (p.283): l'auteur commet toutefois un anachronisme en interprétant les remarques d'Augustin comme une réponse polémique aux «tentatives de récupération que semblaient autoriser certaines versions consensuelles du Roman d'Alexandre», car l'entrée de l'épisode jérusalémite dans la tradition pseudo-callisthénienne n'est pas attestée avant la fin du viII ${ }^{\mathrm{e}}$ ou le début du $\mathrm{IX}^{\mathrm{e}}$ siècle, date de composition de la recension $\varepsilon$.

36 Pour un aperçu cavalier sur la foisonnante diffusion du Roman d'Alexandre, voir l'introduction de G.Bounoure, Pseudo-Callisthène, Le Roman d'Alexandre. La vie et les hauts faits d'Alexandre de Macédoine, en collaboration avec B. Serret, Paris, I992, notamment p. XXXIV-XXXVII.

37 Éd. W. Kroll, Historia Alexandri Magni. Volumen I. Recensio vetusta, Berlin, I926; L. Bergson, Der griechische Alexanderroman. Rezension $\beta$, Stockholm, 1965. 
(XIV ${ }^{\mathrm{e}}-\mathrm{XVI}^{\mathrm{e}} \mathrm{s}$.), et dans la Phyllada néo-grecque (fin $\left.\mathrm{XVII}^{\mathrm{e}} \mathrm{s}.\right)$, adaptation de la précédente recension ${ }^{38}$.

Je passerai rapidement sur le Poème du Marcianus gr. 408, dont l'auteur s'est contenté de reprendre et de versifier le texte de Georges le Moine ${ }^{39}$. La caractéristique la plus remarquable de cette réécriture tardo-byzantine est que l'épisode jérusalémite (v.1604-ı688) ait été interpolé dans un récit qui, dérivant des recensions anciennes $\alpha-\beta$, mettait en scène un héros païen, se mouvant dans un univers à l'antique ${ }^{40}$. Pareil emprunt témoigne assurément de la popularité d'un épisode que le rédacteur a tenu à faire figurer dans son adaptation du Roman, sans trop se préoccuper de savoir s’il était en cohérence avec le reste de l'œuvre.

Les recensions $\varepsilon$ et $\zeta$ sont beaucoup plus intéressantes, dans la mesure où elles offrent une version profondément renouvelée du séjour à Jérusalem. Bien que le texte de la recension $\varepsilon$ présente quelques points communs avec celui de Flavius Josèphe, il n'est pas du tout sûr que son auteur ait utilisé les Antiquités Juives, tant sa version est riche en innovations ${ }^{41}$. On remarquera tout d'abord les modifications apportées à la chronologie des événements : si le séjour d'Alexandre à Jérusalem (ch. 20, 2-5) précède toujours le départ en Égypte, la rencontre avec les prêtres juifs n’a plus lieu sur fond de guerre contre les Perses, car Alexandre,

38 Éd. J. Trumpf, Anonymi byzantini. Vita Alexandri Regis Macedonum, Stuttgart, 1974 (ع); U. von Lauenstein, H. Engelmann et F. Parthe, Der griechische Alexanderroman. Rezensio $\Gamma, 3$ vol., Meisenheim am Glan, I962, I963, Ig69; W.J.Aerts, The Byzantine Alexander Poem, 2 vol., Berlin, 2014 (Marcianus gr. 408); A. Lolos et V.L. Konstantinopoulos, Ps.-Kallisthenes: Zwei mittelgriechische Prosa-Fassungen des Alexanderromans, 2 vol.,

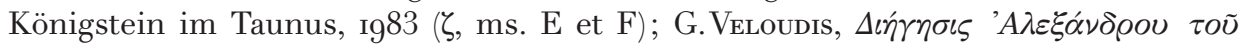

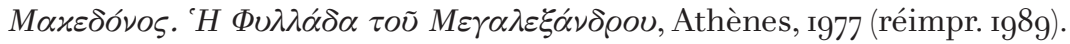

39 Bien que la date de i388, figurant dans le manuscrit, soit souvent considérée comme la date de composition du Poème (cf. C. Matzukis, "The Alexander Romance in the Codex Marcianus 408: New Perspectives for the Date i388, Hellenic Consciousness and Imperial Ideology ", ByzZ 99 [2006], p. Iog-II7), AERTs, partisan d'une chronologie plus haute, suggère de placer la rédaction de ce texte à l'époque de Michel VIII Paléologue, après la reconquête de Constantinople en I26r (The Byzantine Alexander Poem, t. I, p. 6).

40 Cf. C. Jounnno, «Emprunt et réécriture dans une version tardive du Roman d'Alexandre: le poème du Marcianus graecus 408 ", in S. Dusanic et B. Fuusin (éd.), Remanier, métaphraser: fonctions et techniques de la réécriture dans le monde byzantin, Belgrade, 20II, p. 229-238 (p.232-234).

41 Pour une présentation générale de la recension $\varepsilon$, voir C. Jounnno, Naissance et métamorphoses du Roman d'Alexandre. Domaine grec, Paris, 2002, p.339-440; C. Sempéré, La recension $\varepsilon$ du Roman d'Alexandre, traduction et commentaire. L'écriture infinie: le “roman» d'un mythe, thèse de l'université de Montpellier III - Paul Valéry, 20o5, 2 vol.; Ead., "La recension epsilon du Roman d'Alexandre: l'écriture infinie ou le roman d'un mythe », L'Information littéraire 58/4 (2006), p.35-39. 
à son arrivée en Judée, s'est déjà rendu maître de l'empire perse (et Darius est mort). Le comportement initial prêté aux Juifs a, par conséquent, été modifié: ils commencent par se montrer plus agressifs que chez Flavius Josèphe et, prétendant résister à Alexandre, envoient dans le camp macédonien des espions que le Conquérant impressionne par une habile mise en scène, destinée à les convaincre que ses hommes sont prêts à mourir pour lui. Aussi les espions font-ils à leurs compatriotes un rapport de mission si terrifiant que ceux-ci renoncent à leurs résolutions belliqueuses et décident de se rendre à Alexandre. Non attestée par ailleurs, cette péripétie initiale, suggérée peut-être à l'auteur d'ع par l'épisode vétéro-testamentaire des espions envoyés par Moïse au pays de Canaan (Nombres, I3), a pour but de mettre en valeur l'astuce d'Alexandre et la fidélité de ses hommes, et elle donne du peuple juif une image si peu flattée qu'on a pu y voir un trait de polémique anti-judaïque ${ }^{42}$. Dans la suite du récit, l'auteur d' $\varepsilon$ revient toutefois à un scénario beaucoup plus proche de Flavius Josèphe: il décrit le cortège des prêtres juifs s'avançant, en grande pompe, à la rencontre du Conquérant, et celui-ci impressionné, comme l'Alexandre de Josèphe, par le «bel ordonnancement» de la procession ${ }^{43}$. Mais le héros de la recension $\varepsilon$ ne se contente pas de rendre hommage au grand-prêtre et d'offrir un sacrifice à son Dieu : l'auteur du Roman nous fait assister à ce qui est, très littéralement, une scène de conversion du Conquérant au monothéisme. À la profession de foi du grand-prêtre - «Nous servons un dieu unique, qui a créé le ciel, la terre, toutes les choses visibles et invisibles; mais nul homme ne peut exposer sa nature» - Alexandre répond en manifestant le désir d'adorer lui aussi ce Dieu insondable: “Votre dieu sera mon dieu », déclare-t-il aux Juifs, avant de leur promettre la paix, parce qu'ils sont «au service du Dieu vivant ${ }^{44}$ »(ch.20, 4). Autre écart notable par rapport à la version de Flavius Josèphe, la disparition de tous les éléments spécifiquement judaïques qui figuraient dans les Antiquités: l'auteur d'e ne parle pas du Temple et ne fait

42 Pacella, “Alessandro e gli Ebrei », p. ı268-ı269.

43 À la différence de Josèphe, notre auteur n'évoque cependant pas l'étonnement des compagnons d'Alexandre devant l'attitude de leur roi, ni les justifications de ce dernier, alléguant un rêve prophétique: il trouve apparemment fort naturel l'effet produit sur le Macédonien par la vue du grand-prêtre.

44 On remarque la présence, en toute cette séquence, d'une phraséologie où se mêlent échos de l'Ancien et du Nouveau Testament (comme le signale Pfister, “Eine Gründungsgeschichte», p.97). Dans un article consacré aux épisodes à contenu religieux de la recension $\varepsilon$, G. Dehling n'insiste pour sa part que sur les liens d' $\varepsilon$ avec l'Ancien Testament et la tradition judaïque («Alexander der Grosse als Bekenner des jüdischen Gottesglaubens », JSJ I2 (I98I), p. I-5ı ; repris dans Id., Studien zum Frühjudentum. Gesammelte Aufsätze I97I-I987, Göttingen, 2000, p.295-339: p.297-3o7, pour l'épisode jérusalémite). 
pas la moindre référence au Livre de Daniel - omissions peut-être imputables au fait que la recension $\varepsilon$ offre, semble-t-il, en ce passage la réécriture chrétienne d'un texte judaïsant ${ }^{45}$. Ce n'est donc pas, comme chez Flavius Josèphe, la satisfaction qu'inspirent à Alexandre les prédictions de Daniel qui explique sa générosité envers les habitants de Jérusalem ${ }^{46}$, mais son enthousiasme de nouveau converti. L'épisode jérusalémite marque une étape importante dans la biographie du héros d' $\varepsilon$ : Alexandre se mue dès lors en confesseur de la foi. Devenu maître de l'Égypte, il y proclame solennellement la toute-puissance du Dieu unique, “incompréhensible, invisible, insondable, porté sur les Séraphins et glorifié d'une voix trois fois sainte» (ch.24, 2), puis, avec l'aide divine, il enferme aux confins du monde les peuples impurs, Gog et Magog, pour éviter qu'ils ne souillent la terre avant l'heure du Jugement Dernier (ch.39). En christianisant le héros du Roman, l'auteur d' $\varepsilon$ a voulu peindre en lui une figure de basileus exemplaire, conforme aux exigences de l'idéologie impériale byzantine.

La recension $\zeta$, qui dérive d' $\varepsilon$, a poussé le processus encore plus loin, en donnant à Alexandre des allures de roi messianique, dont les armes portent pour emblème les figures du lion, de l'aspic et du basilic, les bêtes foulées aux pieds par le Messie (ch.29, I ${ }^{47}$. Le texte en question a une histoire particulièrement complexe $^{48}$ : sans doute contemporaine des romans Paléologues (XIV ${ }^{\mathrm{e}} \mathrm{s}$.), la version originale, aujourd'hui disparue, a fait l'objet d'une traduction en langue slave, qui fut largement diffusée en Serbie et en Russie; les textes grecs en notre possession, transmis dans des manuscrits dont les plus anciens remontent aux années I520, sont une rétroversion de la traduction slave - va-et-vient linguistique qui atteste la faveur rencontrée par cette réécriture tardo-byzantine des aventures d'Alexandre. L'épisode jérusalémite y occupe une place particulièrement importante (ch.5I-52) - dont témoigne son volume même (plus de i33o mots, au lieu de seulement 400 dans la recension $\varepsilon$ ). Tout en s'inspirant d' $\varepsilon$, l'auteur de $\zeta$ est revenu à un scénario plus proche de Flavius Josèphe (qu'il a sans doute utilisé): l'épisode se situe chez lui, comme dans les Antiquités, sur fond de guerre contre Darius et,

45 Cf. Simon, “Alexandre le Grand Juif et Chrétien».

46 À la fin de l'épisode jérusalémite, il est simplement dit qu'Alexandre refuse les présents en or et en argent apportés par les Juifs, et les consacre à Dieu $(20,5)$. Dans le récit que le héros lui-même fait de son séjour à Jérusalem, dans une lettre à sa mère Olympias (ch. 34, 3), il précise qu'il a fait remise aux Juifs du tribut annuel, adoptant ainsi une politique identique à celle de l'Alexandre de Josèphe ( $A J \mathrm{XI}, 338)$ : ce détail ne figurait pas dans la version narrative du ch. 20.

47 Passage attesté uniquement dans le ms E. Cf. Ps. 9o, I3 (LXX): «Tu marcheras sur l'aspic et le basilic; tu fouleras aux pieds le lion et le serpent ».

48 Cf. U. Moennig, Die spätbyzantinische Rezension * $\zeta$ des Alexanderromans, Cologne, I992. 
si les Juifs commencent par refuser de se soumettre à Alexandre, c'est, comme chez Josèphe, parce qu'ils redoutent les représailles du Grand Roi. L'auteur de $\zeta$ a par ailleurs réintroduit les prophéties de Daniel, auxquelles il donne un relief très remarquable en les mentionnant, avant même l'épisode jérusalémite, lors de la visite d'Alexandre à Rome (ch.44), sorte de doublet anticipatoire du séjour à Jérusalem: ce sont en effet les prêtres de Rome qui, les premiers, rapportent à Alexandre la vision du bouc et du bélier, présage pour le conquérant macédonien de victoire contre les Perses. À Jérusalem, ces prophéties lui sont répétées par un grand-prêtre à qui l'auteur de $\zeta$ a prêté les traits du prophète Jérémie, sans se soucier du double anachronisme généré par un pareil choix, Jérémie étant non seulement bien antérieur à Alexandre, mais antérieur aussi au prophète Daniel, auquel il sert ici de porte-parole ${ }^{49}$. L'intrusion dans le Roman d'Alexandre de cette figure biblique est à mettre en relation avec la prolifération de références vétérotestamentaires à laquelle on assiste dans la recension $\zeta$ : dès les premières lignes de l'épisode jérusalémite, on voit Darius rassembler ses troupes en un lieu, Purgopoia, qui n'est autre que la Tour de Babel, où se produisit «la division des langues » (ch.5o, Io). On note ensuite les rappels d'histoire sainte figurant dans la lettre envoyée par les Juifs à Alexandre - passage de la mer Rouge, exil à Babylone, retour à Jérusalem (ch.5I, 2-3); l'auteur mentionne aussi le Temple de Jérusalem, qu'il appelle «Sion, le Saint des Saints », et dont il prend la peine de préciser qu'il fut édifié par le roi Salomon (ch.52, 2); il évoque enfin, dans la conclusion de l'épisode, toute une série de reliques offertes par les Juifs à Alexandre - pierre ayant orné le casque de Josué, fils de Nahvé; épée de Goliath; armes de Samson; bouclier de Hyopanthos, alias Jonathan, fils du roi Saül (ch.52, Io)...

Bien que, dans cette réécriture intégralement christianisée, l'épisode jérusalémite ne soit pas à proprement parler un épisode de conversion, puisque Alexandre n'est jamais décrit comme un héros païen - dès son arrivée à Jérusalem, il déclare aux Juifs qu'il serait honteux que, servant «le Dieu vivant», ils passent sous la domination des Perses et «ador[ent] les idoles» (ch.5ı, 5) -, l'acte de foi prononcé par le Conquérant en réponse à Jérémie n'en demeure pas moins au cœur de la séquence:

49 On remarquera que, chez Flavius Josèphe lui-même, la référence au livre de Daniel constituait déjà un anachronisme, dans la mesure où cet ouvrage pseudépigraphique fut composé seulement vers i65 av. J.-C., bien après la mort d'Alexandre; mais Josèphe pensait évidemment avoir affaire à un texte écrit par le prophète lui-même, donc remontant au vi siècle av. J.-C., et non à un livre à l'antiquité fallacieuse. Selon FLETCherLouis, une forme antérieure du Livre de Daniel aurait toutefois circulé longtemps avant l'époque de la rédaction définitive, au $\mathrm{II}^{\mathrm{e}} \mathrm{s}$. av. J.-C. (“Alexander the Great's Worship», p.82). 
Vous êtes vraiment - dit-il - les serviteurs du Dieu Très Haut; je crois moi aussi en ce Dieu, je le confesse, l'adore, le célèbre, je lui offre ces présents et [lui remets] le tribut que je voulais vous imposer, ainsi qu'à tous les autres peuples: que votre Dieu soit aussi mon Dieu, et que son amour et sa pitié soient avec moi! (ch.52, 4, ms F)

L'auteur de $\zeta$ s'attarde d'ailleurs à dépeindre son héros en instrument du ToutPuissant, puisqu'il fait dire à Jérémie que «la main du Dieu Sabaoth », désormais, est sur lui $($ ch.52, 6). Annonce confirmée par la suite du récit: après sa visite à Jérusalem, Alexandre vit sous l'emprise permanente du divin, et Jérémie, devenu son protecteur attitré, est constamment présent à ses côtés, pour lui prodiguer conseils et encouragements, l'assurer, par exemple, du soutien du dieu Sabaoth, avant le combat décisif contre Darius ${ }^{50}$, ou, dans les derniers chapitres du Roman, pour l'avertir de sa fin prochaine (ch. I20, I).

On pourrait s'étonner de l'entrée de cette figure vétéro-testamentaire dans la tradition pseudo-callisthénienne, où rien ne semblait imposer sa présence. Peutêtre l'aura dont Jérémie est entouré dans l'œuvre de Flavius Josèphe, qui paraît s'être identifié au prophète qui avait prédit la capitulation de Jérusalem ${ }^{51}$, a-t-elle attisé l'intérêt de l'auteur de $\zeta$ pour le personnage en question, puisqu'il a, selon toute vraisemblance, utilisé le texte des Antiquités juives. La transformation de Jérémie en mentor d'Alexandre fut sans doute facilitée par le lien anciennement établi entre ces deux figures dans la Vie des prophètes, où le transfert des ossements de Jérémie dans la cité d'Alexandrie est attribué au Conquérant ${ }^{52}$. Quant au

50 Il lui suffira d'invoquer «le Dieu unique et saint, créateur du ciel et de la terre, qui se repose sur les Chérubins, Adônaï Sabaoth», pour se rendre maître de tous ceux qui marcheront contre lui (ms E, ch. 55, ı2). Passage manquant dans le ms F.

51 Sur Jérémie comme alter ego de Flavius Josèphe, cf. VIDAL-Naquet, “Flavius Josèphe ou du bon usage de la trahison », p.29; Id., "Flavius Josèphe et les prophètes ”, p. 26; S.J.D. Cohen, “Josephus, Jeremiah, and Polybius», History and Theory 21.3 (1982), p.366-38i (p.367-37o); M. Hadas-Lebel, Flavius Josèphe. Le Juif de Rome, Paris, ig89, p.202-206; W. Den Hollander, Josephus, the Emperors, and the City of Rome: from Hostage to Historian, Leyde - Boston, 2014, p.75, I03, I44, ı66-ı67. M. Tuval se montre plus sceptique concernant l'identification supposée de Josèphe au prophète (From Jerusalem Priest to Roman Jew: on Josephus and the Paradigms of Ancient Judaism, Tübingen, 2013, p. 96, I24 et I86-I88).

52 Vie des prophètes (recension anonyme), trad. M. Petit, in P. Geoltrain et J.-D. Kaestli (éd.), Écrits apocryphes chrétiens, t. II, Paris, 2005, p.419-452 (p.43i): «Nous, nous avons entendu dire par les serviteurs de la maison d'Antigone et de Ptolémée - des hommes âgés - que, lorsqu'Alexandre le Macédonien s'arrêta au tombeau du prophète et en connut les mystères, il fit transporter ses restes à Alexandrie et les déposa solennellement tout autour de la ville. Ainsi fut écartée du pays la race des serpents, comme les crocodiles le furent du fleuve. » Cette tradition concernant la translation des ossements 
rôle joué par le prophète aux côtés d'Alexandre, il rappelle celui tenu par diverses figures de saints hommes auprès des empereurs byzantins ${ }^{53}$. Sans doute peuton interpréter la transformation du conquérant macédonien en figure messianique, dans une œuvre littéraire conçue à une époque où la pression du péril turc menaçait de plus en plus clairement l'existence de l'empire byzantin, comme une réponse aux angoisses du présent: en transformant l'expédition d'Alexandre en une sorte de croisade contre les Infidèles, l'auteur de $\zeta$ fait de lui le héraut de valeurs religieuses menacées par la politique expansionniste de l'empire Ottoman.

\section{Conclusion}

Bien que Flavius Josèphe ait conçu son récit du séjour d’Alexandre à Jérusalem comme une pièce de propagande en faveur du peuple juif, le texte des Antiquités contribuait aussi à légitimer la conquête du roi macédonien, en plaçant son entreprise sous le patronage divin ${ }^{54}$, et c'est ce second aspect de l'épisode que les lecteurs byzantins ont retenu, ainsi que l'idéale collaboration des deux pouvoirs, politique et religieux, à laquelle aboutit la rencontre du Conquérant et du grand-prêtre. Si l'histoire de cette escale jérusalémite suscita un intérêt tout aussi vif chez les lettrés de l'Occident médiéval, où l'épisode est attesté, jusqu'à l'aube de la Renaissance, dans la plupart des domaines linguistiques et, comme à Byzance, aussi bien dans les versions romanesques de la geste d'Alexandre que dans des ouvrages à vocation

de Jérémie est évoquée dans la recension ל en F, ı26, 2-3 (passage absent dans E). Elle était bien connue à Byzance, comme le montre sa présence chez divers chroniqueurs (Chronique Pascale, Zonaras) ou dans la Souda. La faveur dont la figure de Jérémie a bénéficié dans la littérature pseudépigraphique, où il apparaît à la fois comme un nouveau Moïse, à qui Dieu parle face à face, et comme un héraut et une préfiguration du Christ, a sans doute aussi contribué à favoriser son entrée dans le Roman d'Alexandre: cf. J.Riaud, “La figure de Jérémie dans les Paralipomena Jeremiae», in A. Caquot et M. Delcon (éd.), Mélanges bibliques et orientaux en l'honneur de M. Henri Cazelles, Neukirchen - Vluyn, I98I, p.373-385. Sur la diffusion des Paralipomènes de Jérémie dans le domaine slave, voir E. Turdeanu, Apocryphes slaves et roumains de l'Ancien Testament, Leyde, I98I, p. 348-363: l'auteur signale l'existence d'une version serbo-macédonienne, réalisée $\mathrm{au} \mathrm{x}^{\mathrm{e}} \mathrm{ou} \mathrm{xI}{ }^{\mathrm{e}}$ siècle, en Macédoine occidentale ou en Vieille Serbie, et passée très tôt en Russie, peut-être dès le $\mathrm{xI}^{\mathrm{e}}$ siècle.

53 Cf. A. Tiмотіn, Visions, prophéties et pouvoir à Byzance. Étude sur l'hagiographie mésobyzantine, $I X^{e}-X I^{e}$ siècles, Paris, 20I0, p.73-80. La relation privilégiée d'Alexandre avec Jérémie rappelle aussi la dévotion de l'empereur Basile Irr $(867-886)$ pour le prophète Élie (ibid., p. 93-95).

54 Cf. Gruen, Heritage, p. I98; Kıм, “The Dream of Alexander», p.438. 
historiographique ou encyclopédique ${ }^{55}$, aucun texte ne semble être allé aussi loin que ne le firent les recensions byzantines $\varepsilon$ et $\zeta$ dans l'appropriation chrétienne du héros antique: même chez les auteurs qui ont développé le plus amplement le processus d'assimilation d'Alexandre aux valeurs religieuses de l'Occident, le Conquérant demeure dans une sorte d'entre-deux: évoquant l'Alexandre de Jean Wauquelin (vers I/48), qui pourtant a vu le jour dans un contexte fortement marqué par l'imaginaire de la croisade, Catherine Gaullier-Bougassas parle d'une initiation “incomplète» au monothéisme - “conversion à une religion pré-chrétienne ${ }^{56}$ », qui fait pâle figure, face aux professions de foi dépourvues d'ambiguïté du héros, sensi-

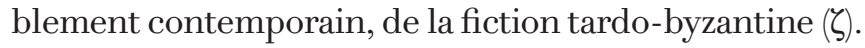

\author{
Corinne Jouanno \\ Université de Caen - Normandie, \\ CRAHAM \\ 144, rue Legendre \\ 75017 Paris \\ corinne.jouanno@unicaen.fr
}

55 Pour un aperçu sur la présence de l'épisode dans les différentes littératures européennes, voir C. Gaullier-Bougassas (éd.), La Fascination pour Alexandre le Grand dans les littératures européennes ( $x^{e}$-XVI siècle), 4 vol., Turnhout, 20I4, t. IV, p. 28, 3I, 36, 43, 72 (littérature latine: Godefroi de Viterbe, Historia de preliis, Quilichinus de Spolète); p. 87, I04, III, II5, I35, I46, I54, I6I, I65, I92, 203, 2I7-218, 224, 235 (littérature de langue française: romans d'Alexandre de Paris et de Thomas de Kent, Alexandre en prose, historiographes comme Wauchier de Denain, Baudoin d'Avesnes, Jean de Vignay, Jean Wauquelin ou Jean Mancel); p. 243, 245, 248, 256, 258, 259, 263 (adaptations italiennes de l'Historia de preliis); p. 269, 279, 283, 287 (littérature hispanique); p.333, 336, 35o, 354, 362, 367 (domaine anglophone); p. 382, 387-388, 409, 426, 432, 437, 449, 45i, 463 (domaine germanique); p.469 et 472 (littérature néerlandaise).

56 C. Gaullier-Bougassas, «Alexandre héros païen ou héros pré-chrétien? Deux stratégies opposées de réécriture à la fin du Moyen Âge », Le Moyen Français 5I-52-53 (2002-2003), p. 3o5-326 (p.3I4 et 3I 7). I. FABre estime pour sa part que, dans le Roman de toute chevalerie de Thomas de Kent, où l'épisode jérusalémite fait pourtant l'objet d'une remarquable amplification, l'initiation d'Alexandre à la “sagesse supérieure » du monothéisme n'a pas lieu («La conversion d'Alexandre le Grand au judaïsme: transpositions et avatars d'une légende dans les Romans d'Alexandre français du xiI ${ }^{\mathrm{e}}$ siècle », Cahiers d'Études du religieux. Recherches interdisciplinaires 7 (2009): article accessible en ligne à l'adresse http://cerri.revues.org/454). L'auteur estime que C. Raynaud a cédé à la tentation de la surinterprétation en analysant comme des images de conversion au christianisme les illustrations de l'épisode jérusalémite figurant dans des manuscrits français des XIII ${ }^{\mathrm{e}}$, $\mathrm{xIV}^{\mathrm{e}}$ et $\mathrm{XV}^{\mathrm{e}}$ siècles (“Alexandre et Jérusalem», in Ead., Mythes, cultures et sociétés (XIII ${ }^{\text {- }}$ $x V^{e}$ s.), Paris, I995, p. 297-322). 


\section{Annexe 1 : Présence de l'épisode d'Alexandre à Jérusalem chez les chroniqueurs byzantins}

\begin{tabular}{|c|c|c|c|}
\hline Chroniqueur & Séjour à Jérusalem & Contexte & Remarques \\
\hline $\begin{array}{l}\text { Eusèbe de Césarée } \\
\text { (vers 26o-339) }\end{array}$ & $\begin{array}{l}\text { oui } \\
\text { Éd. Helm, p. I23 }\end{array}$ & $\begin{array}{l}\text { Entre la prise de } \\
\text { Tyr et le départ en } \\
\text { Égypte }\end{array}$ & Sacrifice \\
\hline $\begin{array}{l}\text { Excerpta } \\
\text { latina Barbari } \\
\text { (traduction latine } \\
\text { d'une chronique } \\
\text { alexandrine } \\
\text { du } v^{\mathrm{e}} \mathrm{s} \text {.) }\end{array}$ & $\begin{array}{l}\text { oui } \\
\text { Éd. Frick, t. I, p. } 270 \\
\text { et 32I-322 }\end{array}$ & $\begin{array}{l}\text { Après la fondation } \\
\text { d'Alexandrie }\end{array}$ & Acte de foi \\
\hline $\begin{array}{l}\text { Jean Malalas } \\
\text { (vers } 49 \mathrm{I}-578 \text { ) }\end{array}$ & non & & \\
\hline $\begin{array}{l}\text { Jean d'Antioche } \\
\text { (début } \text { vII }^{\mathrm{e}} \mathrm{s} \text {.) }\end{array}$ & $\begin{array}{l}\text { Rien dans les } \\
\text { fragments conservés }\end{array}$ & & \\
\hline $\begin{array}{l}\text { Chronique Pascale } \\
\text { (entre 63I et 64I) }\end{array}$ & $\begin{array}{l}\text { oui } \\
\text { Éd. Dindorf, t. I, } \\
\text { p.39o }\end{array}$ & $\begin{array}{l}\text { Après la fondation } \\
\text { d'Alexandrie }\end{array}$ & Prosternation \\
\hline $\begin{array}{l}\text { Georges le Syncelle } \\
\text { († ap. 8Io) }\end{array}$ & $\begin{array}{l}\text { oui } \\
\text { Éd. Mosshammer, } \\
\text { p.3i4 et 3ı8 }\end{array}$ & $\begin{array}{l}\text { Entre la prise de } \\
\text { Tyr et le départ en } \\
\text { Égypte }\end{array}$ & $\begin{array}{l}\text { Même version } \\
\text { qu'Eusèbe } \\
\text { Sacrifice }\end{array}$ \\
\hline $\begin{array}{l}\text { Georges le Moine } \\
\left(\mathrm{Ix}^{\mathrm{e}} \mathrm{s} .\right)\end{array}$ & $\begin{array}{l}\text { oui } \\
\text { Éd. de Boor, t. I, } \\
\text { p.26-32 }\end{array}$ & $\begin{array}{l}\text { Épisode inséré } \\
\text { dans le récit de la } \\
\text { campagne contre } \\
\text { Darius, entre la prise } \\
\text { de Tyr et la bataille } \\
\text { d'Issos }\end{array}$ & $\begin{array}{l}\text { Reprise de Flavius } \\
\text { Josèphe } \\
\text { Excursus sur le } \\
\text { costume du grand } \\
\text { prêtre } \\
\text { Prosternation + } \\
\text { sacrifice } \\
\text { Épisode de } \\
\text { Mosomachos }\end{array}$ \\
\hline $\begin{array}{l}\text { Syméon Magistros } \\
\left(\mathrm{x}^{\mathrm{e}} \mathrm{s} .\right)\end{array}$ & $\begin{array}{l}\text { oui } \\
\text { Éd. Bekker, p.49 }\end{array}$ & $\begin{array}{l}\text { Épisode inséré } \\
\text { dans le récit de la } \\
\text { campagne contre } \\
\text { Darius, après la prise } \\
\text { de Tyr }\end{array}$ & Prosternation \\
\hline $\begin{array}{l}\text { Georges Kédrénos } \\
\left(\mathrm{XI}^{\mathrm{e}}-\mathrm{XII}^{\mathrm{e}} \mathrm{s} .\right)\end{array}$ & $\begin{array}{l}\text { oui } \\
\text { Éd. Bekker, t. I, p. } 265 \\
\text { et 27I-272 }\end{array}$ & $\begin{array}{l}\text { Entre la prise de Tyr } \\
\text { et de Sidon et celle } \\
\text { de Gaza - avant le } \\
\text { séjour en Égypte }\end{array}$ & $\begin{array}{l}\text { Sacrifice } \\
\text { Épisode de } \\
\text { Mosomachos }\end{array}$ \\
\hline
\end{tabular}




\begin{tabular}{|c|c|c|c|}
\hline $\begin{array}{l}\text { Jean Zonaras } \\
(† \text { ap. II59) }\end{array}$ & $\begin{array}{l}\text { oui } \\
\text { Éd. Pinder, t. I, p. } 329 \\
\text { et 353-355 }\end{array}$ & $\begin{array}{l}\text { Après la prise de Tyr } \\
\text { Récit placé en } \\
\text { appendice, à la fin } \\
\text { du chapitre sur } \\
\text { Alexandre }\end{array}$ & $\begin{array}{l}\text { Version légèrement } \\
\text { abrégée de Flavius } \\
\text { Josèphe } \\
\text { Prosternation + } \\
\text { sacrifice } \\
\text { Démêlés des Juifs et } \\
\text { des Samaritains }\end{array}$ \\
\hline $\begin{array}{l}\text { Constantin } \\
\text { Manassès (vers II3o } \\
\text { - II87) }\end{array}$ & non & & \\
\hline $\begin{array}{l}\text { Michel Glycas } \\
\left(\mathrm{I}^{\text {er }} \text { tiers XII }{ }^{\mathrm{e}} \mathrm{s} .-\dagger \text { peu }\right. \\
\text { apr. I204) }\end{array}$ & $\begin{array}{l}\text { oui } \\
\text { Éd. Bekker, p. } 267\end{array}$ & $\begin{array}{l}\text { Épisode inséré } \\
\text { dans le récit de la } \\
\text { campagne contre } \\
\text { Darius }\end{array}$ & $\begin{array}{l}\text { Prosternation + } \\
\text { sacrifice }\end{array}$ \\
\hline 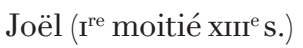 & non & & \\
\hline
\end{tabular}

\section{Annexe 2: Présence de l'épisode d'Alexandre à Jérusalem dans les différentes versions grecques du Roman d'Alexandre}

\begin{tabular}{|c|c|c|c|}
\hline Version du $R A$ & Séjour à Jérusalem & Contexte & Source \\
\hline $\begin{array}{l}\text { Recension } \alpha \text { (entre } \\
\text { la fin de l'époque } \\
\text { hellénistique et le } \\
\text { III }^{\mathrm{e}} \text { s. apr. J.-C.) }\end{array}$ & non & & \\
\hline Recension $\beta\left(v^{e} s.\right)$ & non & & \\
\hline $\begin{array}{l}\text { Recension } \lambda \text { (entre le } \\
\left.\text { vıI }^{\mathrm{e}} \text { et le } \mathrm{x}^{\mathrm{e}} \mathrm{s} .\right)\end{array}$ & non & & \\
\hline $\begin{array}{l}\text { Recension } \varepsilon\left(\text { fin } \mathrm{VIII}^{\mathrm{e}}-\right. \\
\left.\text { début } \mathrm{IX}^{\mathrm{e}} \mathrm{s} .\right)\end{array}$ & $\begin{array}{l}\text { oui } \\
\text { Éd. Trumpf, ch. } 20\end{array}$ & $\begin{array}{l}\text { Après la mort de } \\
\text { Darius } \\
\text { Avant le séjour en } \\
\text { Égypte }\end{array}$ & \\
\hline $\begin{array}{l}\text { Recension } \gamma \text { (entre le } \\
\left.\mathrm{Ix}^{\mathrm{e}} \text { et le } \mathrm{xIV}^{\mathrm{e}} \mathrm{s} .\right)\end{array}$ & $\begin{array}{l}\text { oui } \\
\text { Éd. von Lauenstein, } \\
\text { Engelmann et } \\
\text { Parthe, l. II, ch. } 24\end{array}$ & $\begin{array}{l}\text { Après la mort de } \\
\text { Darius } \\
\text { Avant le second } \\
\text { séjour d'Alexandre } \\
\text { en Égypte }\end{array}$ & Recension $\varepsilon$ \\
\hline
\end{tabular}




\begin{tabular}{|c|c|c|c|}
\hline $\begin{array}{l}\text { Marcianus gr. } 408 \\
\text { (I388?) }\end{array}$ & $\begin{array}{l}\text { oui } \\
\text { Éd. Aerts, } \\
\text { v. I604-ı688 }\end{array}$ & $\begin{array}{l}\text { Après le séjour en } \\
\text { Égypte } \\
\text { Épisode inséré } \\
\text { dans le récit de la } \\
\text { campagne contre } \\
\text { Darius, après la prise } \\
\text { de Tyr }\end{array}$ & Georges le Moine \\
\hline $\begin{array}{l}\text { Recension } \zeta \\
\text { (original du } \mathrm{XIV}^{\mathrm{e}} \mathrm{s} . \text { ? } \\
\text { rétroversion grecque } \\
\mathrm{Xv}^{\mathrm{e}} / \text { début } \mathrm{xvI}^{\mathrm{e}} \mathrm{s} \text {.) }\end{array}$ & $\begin{array}{l}\text { oui } \\
\text { Éd. Lolos et } \\
\text { Konstantinopoulos } \\
\text { ch. 5o, II - ch. 52 }\end{array}$ & $\begin{array}{l}\text { Épisode inséré } \\
\text { dans le récit de la } \\
\text { campagne contre } \\
\text { Darius, avant le } \\
\text { départ pour l'Égypte }\end{array}$ & $\begin{array}{l}\text { Recension } \varepsilon \\
+ \text { Flavius Josèphe }\end{array}$ \\
\hline $\begin{array}{l}\text { Rimada (éditée en } \\
\text { I529) }\end{array}$ & non & & \\
\hline Phyllada (fin XvıI' ${ }^{\mathrm{e}} \mathrm{s}$ ) & $\begin{array}{l}\text { oui } \\
\text { Éd. Veloudis, } \\
\S 9^{6-\text { Io5 }}\end{array}$ & $\begin{array}{l}\text { Épisode inséré } \\
\text { dans le récit de la } \\
\text { campagne contre } \\
\text { Darius, avant le } \\
\text { départ pour l'Égypte }\end{array}$ & Recension $\zeta$ \\
\hline
\end{tabular}

